\title{
Estimation of damping in layered welded structures with unequal thickness
}

\author{
Bhagat Singh* and Bijoy Kumar Nanda \\ Department of Mechanical Engineering, National Institute of Technology, Rourkela, Odisha, India
}

Received 28 March 2011

Revised 5 February 2012

\begin{abstract}
The present work is focused on the study of damping mechanism in layered and welded cantilever beams with unequal thickness. It is observed that a number of vital parameters such as: thickness ratio, pressure distribution characteristics, relative slip and kinematic co-efficient of friction at the interfaces, initial amplitude of excitation, length and thickness of the beam specimen govern the damping capacity of these structures. Experiments have been conducted in order to study the effect of these parameters and validate the developed damping model. The damping model of the structure is found to be in fairly good agreement with the measured data. Finally, the results for the damping capacity are discussed and rationalized.
\end{abstract}

Keywords: Slip damping, tack welded joint, amplitude, unequal thickness, in-plane bending stress

\section{Introduction}

Built-up structures exhibit vibration damping due to slip at the joints. Although, numerous works have been carried out on this aspect, still the mechanism of damping in fabricated structures remains one of the least well-understood subjects for general vibration analysis. The major reason attributed for this is the absence of a universal mathematical model to represent the damping forces. In many modern automotive, machine tool and aircraft industries, welded joints are mostly used to assemble the parts. These joints have a great potential to reduce the vibration levels of these structures, thereby attracting the interest of many researchers to understand the mechanism of vibration attenuation. In an earlier attempt, Goodman and Klumpp [1] examined the energy dissipation due to slip at the interfaces of a laminated beam. In fact, previous investigators such as; Cockerham and Symmons [2], Hess et al. [3] and Guyan et al. [4] developed various friction and excitation models, while Barnett et al. [5] and Maugin et al. [6] considered interfacial slip waves between two surfaces for the measurement of damping capacity of structures.

Studies by researchers such as; Goodman [7], Earles [8], Murty [9] have shown that the energy dissipation at the joints occur due to frictional energy loss at the interfaces, which is more than the energy loss at the support. In fact following the work of Goodman and Klumpp [1], early workers, such as Masuko et al. [10], Nishiwaki et al. [11], and Motosh [12] studied the damping capacity of layered and bolted structures assuming uniform intensity of pressure distribution at the interfaces of these structures. However, their work is limited to the layered and jointed symmetric structures.

Hansen and Spies [13] investigated the structural damping in laminated beams due to interfacial slip. They analyzed a two layered plate model with an assumption that there exists an adhesive layer of negligible thickness and mass between the two layers such that some amount of micro-slip originates at the frictional interfaces which

*Corresponding author: Bhagat Singh, Department of Mechanical Engineering, National Institute of Technology, Rourkela, Odisha, India. Tel.: +91 7742989240; E-mail:bhagatmech@gmail.com. 


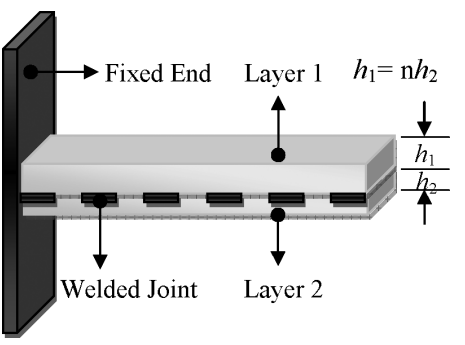

(a)

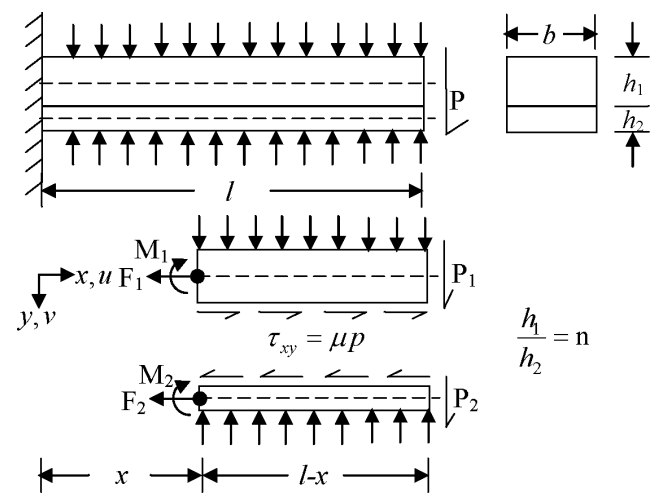

(b)

Fig. 1. (a) Two layered tack welded cantilever beam model; (b) Two halves of the beam depicting load and co-ordinates.

contributes to the damping. They have also shown that the restoring force is developed by this adhesive medium and is proportional to the interfacial micro-slip.

Following the work of Masuko et al. [10], Nanda and Behera [14] examined the interfacial slip damping in multilayered bolted structures and developed a theoretical expression for the pressure distribution at the interfaces of a bolted joint by curve fitting the earlier data reported by Ziada and Abd [15]. Nanda and Behera [16,17] and Nanda [18] further studied the distribution pattern of the interface pressure as well as the damping capacity of layered and jointed structures by carrying out both numerical analysis and experiments to ascertain the effects of number of layers, diameter of bolts and use of washers. However, they neglected the effect of in-plane bending stresses on the dynamic slip and damping capacity. Moreover, their work was limited to layered and bolted structures with symmetric cantilever beams.

Recently, Damisa et al. [19] examined the effect of non-uniform interface pressure distribution on the mechanism of slip damping in layered beams, but their analysis is limited to the case of static load. Damisa et al. [20] also examined the effect of non-uniform interface pressure distribution on the mechanism of slip damping for layered beams under dynamic loads. Though these researchers considered the in-plane distribution of bending stresses but all the analysis is limited to the symmetric structures with single interface.

A lot of work has been carried out on the damping capacity of bolted structures but little work has been reported on the mechanism of damping in layered and welded structures. Recently, Singh and Nanda [21] proposed a method to evaluate the damping capacity of tack welded structures and established that the damping capacity decreases with the increase in the number of tack welds.

Research works reported till date is silent on the mechanism of damping in layered and welded beams of unequal thickness. The present work is devoted on the development of a mathematical model, capable of accurately incorporating the effects of welded joints on the damping capacity of layered and welded structure of unequal thickness. Moreover, the damping capacity of un-symmetric structure has been evaluated theoretically and validated experimentally. Further, the findings are compared with that of the equivalent welded symmetrical beams. Qualitative trends are identified in the measurements and the suitability of capturing these effects is addressed.

\section{Theoretical analysis}

The tack welded two layered cantilever beam model with overall thickness $2 h$, width $b$, and length $l$ as shown in Fig. 1(a) is considered to evaluate the damping ratio. The loading consists of uniformly distributed pressure at the interfaces due to perfect contact between two flat bodies, and a concentrated load $P$ applied at the free end, $x=l$. Each of the two halves of thickness $h_{1}$ and $h_{2}$ is considered separately with the loading as depicted in the Fig. 1(b). The continuity of stress and vertical displacement ' $v$ ' is imposed at the interfaces. At some finite value of $P$, the shear stress at the interfaces will reach the critical value for slip $\tau_{x y}=\mu p$, where $\mu$ and $p$ are the kinematic co-efficient friction and interface pressure respectively. Additional static force due to excitation will produce a relative displacement $\Delta u(x)$ at the interfaces. 


\subsection{Interface pressure distribution}

The contact pressure for flat surfaces with rounded corners has been found out by Ciavarella et al. [22], which shows a non-uniform distribution pattern at the interfaces. Contrary to this, the pressure distribution at the interfaces is assumed to be uniform owing to the contact of the upper layer over the lower one. Therefore, the relation for uniform pressure distribution as given by Johnson [23] and Giannakopoulos et al. [24] due to contact of two flat bodies has been considered and the same is given by:

$$
p(x)=\frac{W}{b}
$$

where $W$ and $b$ are the normal load per unit length and width of the beam respectively.

\subsection{Analysis of static response}

The static response is evaluated considering the layered and welded cantilever beams of unequal thickness. The ratio of the two layers considered is given by:

$$
\frac{h_{1}}{h_{2}}=n
$$

where $h_{1}, h_{2}$ are the thickness of the upper and lower layers respectively and $n$ is a constant.

Furthermore,

$$
h_{1}+h_{2}=2 h
$$

Solving expressions (2) and (3) thickness of the upper and lower layers is given by:

$$
\begin{aligned}
& h_{1}=2 h n / n+1 \\
& h_{2}=2 h / n+1
\end{aligned}
$$

The resultant moment at the centroid of each laminate as shown in Fig. 1 (b) is given by:

$$
\begin{aligned}
& M_{1}=P_{1}(l-x)-\mu p b \frac{n h}{n+1}(l-x) \\
& M_{2}=P_{2}(l-x)-\mu p b \frac{h}{n+1}(l-x)
\end{aligned}
$$

where the subscripts 1 and 2 refer to the upper and lower laminates respectively. Moreover, $P_{1}$ and $P_{2}$ are the static forces acting on the laminates 1 and 2 respectively as shown in Fig. 1(b).

Furthermore,

$$
P_{1}+P_{2}=P
$$

Invoking the relation between bending moment and curvature as derived by Warburton [25], we get;

$$
M=-E I \frac{d^{2} v}{d x^{2}}
$$

where $E$ and $I$ are the modulus of elasticity and moment of inertia of the welded beam cross-section, respectively.

Putting expression (5) in (7) the following expression is obtained;

$$
\begin{aligned}
& \frac{d^{2} v_{1}}{d x^{2}}=\frac{1}{E I_{1}}\left(P_{1}-\frac{\mu p b n h}{n+1}\right)(l-x) \\
& \frac{d^{2} v_{2}}{d x^{2}}=\frac{1}{E I_{2}}\left(P_{2}-\frac{\mu p b h}{n+1}\right)(l-x)
\end{aligned}
$$


where $I_{1}\left(b h_{1}^{3} / 12\right), I_{2}\left(b h_{2}^{3} / 12\right), v_{1}$ and $v_{2}$ are the moment of inertia and static response of the laminates 1 and 2 respectively.

Integrating expression (8) once we get;

$$
\begin{aligned}
& \frac{d v_{1}}{d x}=\frac{1}{E I_{1}}\left(P_{1}-\frac{\mu p b n h}{n+1}\right)\left(l x-\frac{x^{2}}{2}\right)+C_{1 a} \\
& \frac{d v_{2}}{d x}=\frac{1}{E I_{2}}\left(P_{2}-\frac{\mu p b h}{n+1}\right)\left(l x-\frac{x^{2}}{2}\right)+C_{1 b}
\end{aligned}
$$

where $C_{1 a}, C_{1 b}$ are the integration constant and is evaluated to be zero by putting the boundary condition, $\left.\left(d v_{1} / d x\right)\right|_{x=0}=\left.\left(d v_{2} / d x\right)\right|_{x=0}=0$ in the expression (9).

Further, integration of expression (9) yields;

$$
\begin{aligned}
& v_{1}=\frac{3(n+1)^{3}}{2 E b h^{3} n^{3}}\left(P_{1}-\frac{\mu p b h n}{n+1}\right)\left(\frac{l x^{2}}{2}-\frac{x^{3}}{6}\right)+C_{2 a} \\
& v_{2}=\frac{3(n+1)^{3}}{2 E b h^{3}}\left(P_{2}-\frac{\mu p b h}{n+1}\right)\left(\frac{l x^{2}}{2}-\frac{x^{3}}{6}\right)+C_{2 b}
\end{aligned}
$$

where the integration constants, $C_{2 a}=C_{2 b}=0$ since $\left.v_{1}\right|_{x=0}=\left.v_{2}\right|_{x=0}=0$

Furthermore, it is assumed that the continuity equation prevails as such;

$$
v_{1}=v_{2}
$$

Solving expressions (10) and (11) we get;

$$
\begin{aligned}
& P_{1}=\frac{n^{3} P+n\left(2 n^{3}-n+1\right) Q}{n^{3}+1} \\
& P_{2}=\frac{P+n(n-1) Q}{n^{3}+1}
\end{aligned}
$$

The two dimensional parameters $\mathrm{Q}$ and $\mathrm{R}$ are defined as;

$$
\begin{aligned}
& Q=\mu p b h \\
& R=\frac{E b h^{3}}{l^{3}}
\end{aligned}
$$

Using the expressions (10), (12) and (13), the static response in terms of "Q" and "R" are finally found to be;

$$
v_{1}=v_{2}=\frac{(n+1)^{3}}{4 R\left(n^{3}+1\right)}(P-Q)\left[3\left(\frac{x}{l}\right)^{2}-\left(\frac{x}{l}\right)^{3}\right]
$$

\subsection{Evaluation of relative dynamic slip}

The displacement at any axial position $x$ and $y_{1,2}=\mp h / 2$ are given by:

$$
\begin{aligned}
& u_{1}=\frac{1}{E} \int_{0}^{x} \sigma_{x_{1}} d x-\frac{h_{1}}{2} \frac{d v_{1}}{d x} \\
& u_{2}=\frac{1}{E} \int_{0}^{x} \sigma_{x_{2}} d x+\frac{h_{2}}{2} \frac{d v_{2}}{d x}
\end{aligned}
$$


These displacements are produced by the resultant axial force $F_{1,2}$ and moment $M_{1,2}$, about the centroid of each half of the beam as shown in Fig. 1 (b).

where $v_{1}, v_{2}$ are the vertical deflections, $E$ is the modulus of elasticity and $\sigma_{x_{1}}, \sigma_{x_{2}}$ are the in-plane bending stresses. It is assumed that the continuity equation prevails, i.e., $v_{1}=v_{2}=v$.

From the force equilibrium, the in-plane bending stresses in the upper and lower laminates are computed as:

$$
\begin{aligned}
\sigma_{x_{1}} & =\frac{\mu p(n+1)}{2 n h}(l-x) \\
\sigma_{x_{2}} & =-\frac{\mu p(n+1)}{2 h}(l-x)
\end{aligned}
$$

Combining expressions (14), (15) and (16) and simplifying, the relative slip displacement at the interfaces is given by:

$$
\Delta u=u_{2}-u_{1}=\frac{3(n+1)^{3} h}{4\left(n^{3}+1\right) R l}\left[P-\frac{\left(n^{3}+3 n^{2}+3 n+1\right)}{3 n(n+1)} Q\right]\left[2\left(\frac{x}{l}\right)-\left(\frac{x}{l}\right)^{2}\right]
$$

Slip will occur only if $P>P_{c}=\frac{\left(n^{3}+3 n^{2}+3 n+1\right)}{3 n(n+1)} Q$.

where, $P_{c}$ is the critical static load applied at the tip of the welded cantilever beams.

\subsection{Analysis of energy dissipation}

The energy is dissipated due to friction and relative dynamic slip at the interfaces. For completely reversed loading, the product of the shear force, $\mu p$ and the relative displacement, $\Delta u$ are integrated over the length of the beam and is found to be equal to one-fourth of the energy dissipated in a complete cycle.

Thus, energy dissipation per cycle as established by Goodman and Klumpp [1] is given by:

$$
E_{\text {loss }}=4 b \int_{0}^{l} \tau_{x y} \Delta u(x) d x=4 \mu p b \int_{0}^{l}\left(u_{2}-u_{1}\right) d x
$$

where, $u_{1}$ and $u_{2}$ are the displacements in the x-direction of points on the adjacent faces of the upper and lower half beam respectively.

Substituting the expression (17) in (18) considering the beam to be loaded cyclically between the loads $\pm P_{m}$ and integrating, the energy dissipation per cycle in terms of static load is given by:

$$
E_{\text {loss }}=\frac{2(n+1)^{3} Q}{\left(n^{3}+1\right) R}\left(P_{m}-\frac{\left(n^{3}+3 n^{2}+3 n+1\right) Q}{3 n(n+1)}\right)
$$

where, $P_{m}$ is the maximum static load applied at the tip of the welded cantilever beams.

The maximum tip displacement $v_{m}$, corresponding to $P=P_{m}$ is obtained from Eq. (14) by putting $x=l$ as follows;

$$
v_{m}=\frac{(n+1)^{3}}{2\left(n^{3}+1\right) R}\left(P_{m}-Q\right)
$$

On rearranging expression (20a), $P_{m}$ in terms of $v_{m}$ is given by:

$$
P_{m}=\frac{2\left(n^{3}+1\right) R v_{m}}{(n+1)^{3}}+Q
$$

Putting the expression (20b) in (19), the energy dissipation in terms of displacement is given by:

$$
E_{l o s s}=4 Q\left(v_{m}-\frac{(n+1)^{2}}{6 n} \frac{Q}{R}\right)
$$

From the expression (19), it is evident that the slip will occur only if $v=v_{c}=\frac{(n+1)^{2}}{6 n} \frac{Q}{R}$, where, $v_{c}$ is the critical tip displacement. 


\subsection{Evaluation of damping ratio}

In vibration problems, it is most convenient to express the dissipative properties of the system in terms of non-dimensional quantities such as the damping ratio $(\psi)$ and loss factor $\left(\eta_{s}\right)$, defined by:

$$
\begin{aligned}
& \psi=\frac{E_{\text {loss }}}{E_{n e}} \\
& \eta_{s}=\frac{E_{\text {loss }}}{2 \pi E_{n e}}=\frac{\psi}{2 \pi}
\end{aligned}
$$

where, $E_{n e}$ is the maximum strain energy stored in the system.

The maximum strain energy stored in the system in terms of maximum load and tip deflection is given by:

$$
E_{n e}=\frac{1}{2} P_{m}\left(\frac{v_{c}}{P_{c}}\right) P_{m}=\frac{P_{m}^{2}}{4 R}
$$

Inserting expression (20b) in (23a) and simplifying we get;

$$
E_{n e}=\frac{1}{4 R}\left(\frac{2\left(n^{3}+1\right) R v_{m}}{(n+1)^{3}}+Q\right)^{2}
$$

Putting expressions (21) and (22) in expression (23) and simplifying, the damping ratio in terms of tip displacement and load is given by:

$$
\psi=\frac{16 Q}{R} \frac{\left[v_{m}-\frac{(n+1)^{2}}{6 n}\left(\frac{Q}{R}\right)\right]}{\left[\frac{2\left(n^{3}+1\right) R v_{m}}{(n+1)^{3}}+Q\right]^{2}}
$$

Putting expression (24) in (22) the expression for loss factor for two layered welded structures with unequal thickness is given by:

$$
\eta_{s}=\frac{8 Q}{\pi R} \frac{\left[v_{m}-\frac{(n+1)^{2}}{6 n}\left(\frac{Q}{R}\right)\right]}{\left[\frac{2\left(n^{3}+1\right) R v_{m}}{(n+1)^{3}}+Q\right]^{2}}
$$

\section{Experimental setup and experiments}

Experiments have been conducted to validate the developed mathematical model for the loss factor of layered and tack welded mild steel beams of unequal thickness. An experimental set-up as shown in Fig. 2 has been fabricated to conduct the experiments. The specimens are prepared from the stock of mild steel flats by tack welding two layers of various thickness and cantilever length. The details of the mild steel specimen used for experimentation are given in Table 1. Experiments have been performed to analyze the influence of contact pressure developed at the interfaces by distributed tack welded joints on the damping of layered and tack welded un-symmetric beams. Experimental loss factor have been estimated using the half-power band width method. The cantilever specimens are excited transversally at the amplitudes of $0.1,0.2,0.3,0.4$ and $0.5 \mathrm{~mm}$ at their free ends with the help of an exciter. The input excitation and output vibration are sensed with vibration pick-ups and the corresponding signal is fed to a digital storage oscilloscope which is connected to the computer with vibration analyzer software i.e., Lab View of National Instruments limited.

The acquired input and output amplitude signals are analyzed using the vibration analyzer software. The frequency response function (FRF) has been generated using the measured amplitudes of input and output signals. The damping ratio is evaluated from the frequency spectrum generated using the experimental data. To estimate damping ratio from frequency domain, half-power bandwidth method has been used. In this method, FRF amplitude of the system is obtained first. Corresponding to each natural frequency, peak in frequency spectrum at resonance condition is 
Table 1

Details of mild steel specimens used for layered and jointed beams

\begin{tabular}{cccc}
\hline $\begin{array}{c}\text { Thickness of the } \\
\text { specimen }\left(h_{1}+h_{2}\right) \\
(\mathrm{mm})\end{array}$ & $\begin{array}{c}\text { Width of the } \\
\text { specimen } \\
(\mathrm{mm})\end{array}$ & $\begin{array}{c}\text { Cantilever } \\
\text { length } \\
(\mathrm{mm})\end{array}$ & $\begin{array}{c}\text { Type of } \\
\text { welding }\end{array}$ \\
\hline$(6+6)$ & 40.2 & 520.6 & Tack Welding \\
$(8+4)$ & 40.2 & & \\
$(9+3)$ & 40.2 & & \\
$(10+2)$ & 40.2 & & \\
$(6+6)$ & 40.2 & 560.4 & Tack Welding \\
$(8+4)$ & 40.2 & & \\
$(9+3)$ & 40.2 & & \\
$(10+2)$ & 40.2 & & \\
$(6+6)$ & 40.2 & 600.6 & Tack Welding \\
$(8+4)$ & 40.2 & & \\
$(9+3)$ & 40.2 & & \\
$(10+2)$ & 40.2 & &
\end{tabular}

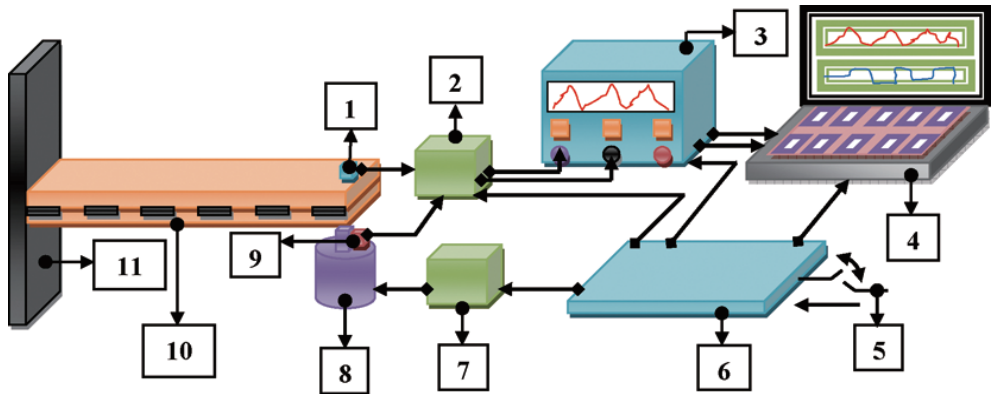
1. Output Vibration Pickup
2. Amplifier
3. Vibration Acquisition
4. Vibration Analyzer
5. Power Supply
6. Distribution Box
7. Power Amplifier
8. Vibration Generator 9. Input Excitation Pickup
10. Tack Welded Beam
11. Fixed End

Fig. 2. Experimental setup.

observed. The maximum dynamic displacement $\left(X_{\max }\right)$ at resonance and resonant frequency $\left(\omega_{n}\right)$ is located in the frequency spectrum, the half-power points are determined when the amplitude is $X_{\max } / \sqrt{2}$ and the corresponding frequencies on either side of the resonant frequencies, $\omega_{1}$ and $\omega_{2}$ are also determined as shown in Fig. 3 . It is observed that at the lower amplitudes of excitation frequency range is more between these two points. Half-power bandwidth is defined as the ratio of the frequency range between the two half power points to the natural frequency at this mode. The damping ratio is then evaluated using the half-power bandwidth expression as given by:

$$
\psi=\frac{\omega_{2}-\omega_{1}}{2 \omega_{n}}
$$

The loss factor is evaluated using the damping ratio and is given by:

$$
\eta_{s}=\frac{\psi}{2 \pi}
$$

The damping ratio measurements are recorded using the storage oscilloscope and laptop interface for welded specimens. The test specimens are fixed at one end with the help of mechanical vice as shown in Fig. 2. Proper care has been taken to ensure the perfect cantilever condition. The vibration generator is placed at the driving point just below the free end of the cantilever specimen. This shaker is used to excite the structure via a function generator with excitations in a frequency range from 0 to $500 \mathrm{~Hz}$. The signal has been amplified by a power amplifier before being fed to the vibration exciter. The natural frequency is also measured under the excitation from the function 


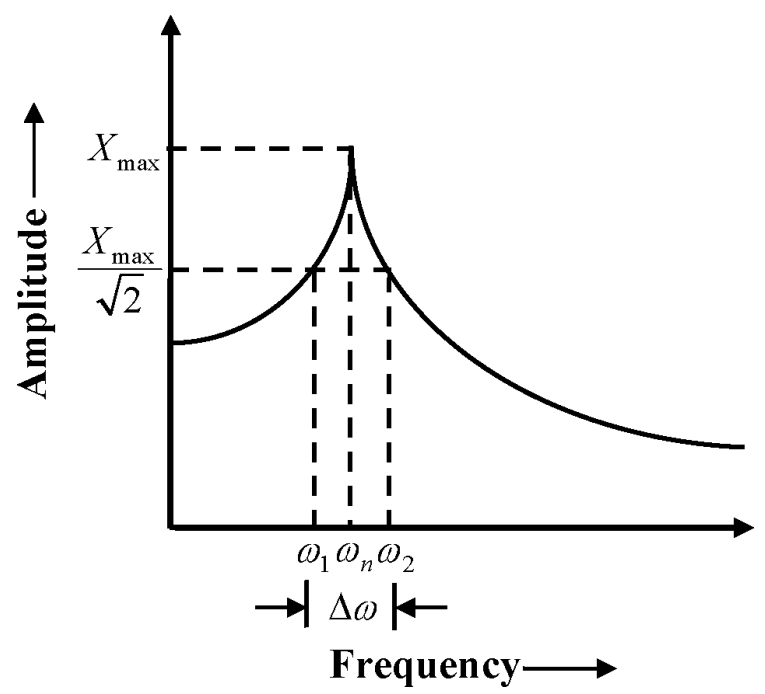

Fig. 3. Half-power bandwidth method for damping measurement.

generator at the point of resonance. The response is measured with an accelerometer which is placed normal to the surface at a specific point i.e., at the free end of the welded cantilever beams. The storage oscilloscope has been setup to measure averages of 25 readings within the frequency range of 0 to $500 \mathrm{~Hz}$, in order to measure the input (channel 1) and output (channel 2) signals. The acquired input and the output signal are transmitted to the computer using the visa software and USB channel via amplifier. The amplifier is used to amplify the low frequency response. The Lab View software is used to analyze the acquired vibration signals. After connecting the set-up along with the analyzer, the start button on the analyzer is pressed in order to start the averaging of the 25 readings. After finding out the averages, FRF curves are generated and the data is saved in a storage drive. FFT based measurements are subject to errors because of an effect known as leakage. Appropriate window function has been applied to correct this problem. The window reduces the leakage and provides more accurate amplitude measurements for the resonant frequencies. This process is repeated for the various specimens and the corresponding experimental results are recorded. The frequency response curves at different amplitudes of excitation loadings have been generated. Some of the frequency response curves obtained at various initial amplitudes of excitation is shown in Fig. 4. The experimental damping ratio is then evaluated using the expression (27) and experimentally generated FRF curves. Experiments are performed to evaluate the experimental loss factors for different mild steel specimens. These experimental results are compared with the theoretical ones and the detailed discussions are presented in the succeeding section.

\section{Results and discussion}

The loss factor of two layered and tack welded mild steel specimens of unequal thickness have been found out numerically using the expression (25). From the analysis following inferences are drawn as detailed below.

Energy loss in welded structures with various thickness ratios for a particular configuration has been plotted in the Fig. 5. All other parameters remaining constant, initiation of slip requires a larger displacement for a thickness ratio greater than one and the energy loss is lower compared to the welded beams of equal thickness. This signifies that the energy dissipation is maximized by having the slip interface at the centroid of the total beam cross-section. The variation of critical load and amplitude has been plotted in Fig. 6. From the plots it is evident that the critical load and amplitude of vibration increases with the increase in thickness ratio. Slip interface is shifted away from the centroid of the total beam cross-section with the increase in thickness ratio, hence greater critical load is required to initiate the slip at the interface. The variation of relative slip with an axial distance from the fixed end has been plotted in Fig. 7. From the figure it is quite evident that the relative slip increases with the distance from the fixed end. 


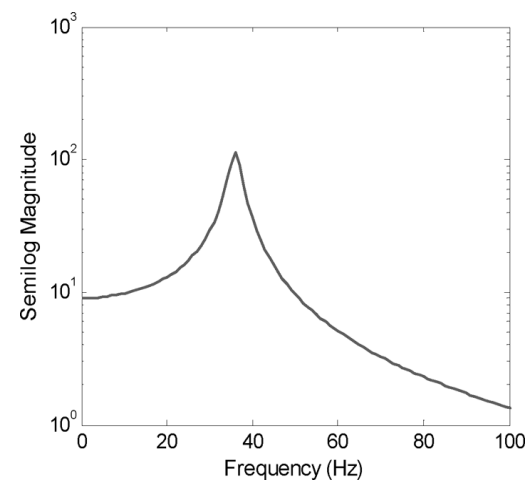

(a)

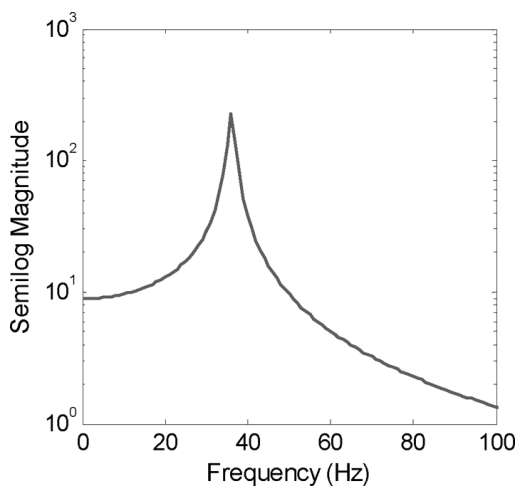

(b)

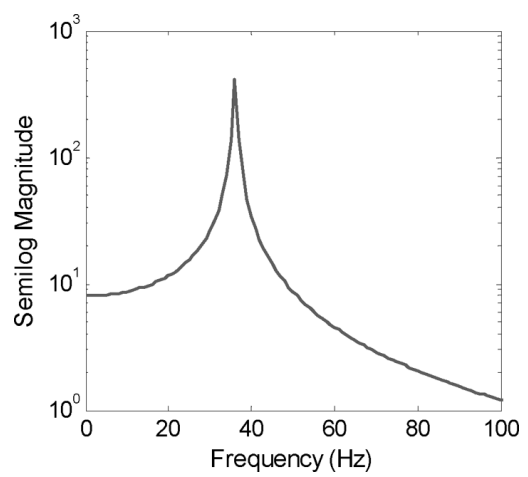

(c)

Fig. 4. (a) FRF plot at $0.1 \mathrm{~mm}$ initial amplitude of excitation for jointed and welded cantilever beams of dimensions $=600.6 \times 40.2 \times 6 \mathrm{~mm}^{3}$; (b) FRF plot at $0.2 \mathrm{~mm}$ initial amplitude of excitation for jointed and welded cantilever beams of dimensions $=600.6 \times 40.2 \times 6 \mathrm{~mm}^{3}$; $(\mathrm{c})$ FRF plot at $0.3 \mathrm{~mm}$ initial amplitude of excitation for jointed and welded cantilever beams of dimensions $=600.6 \times 40.2 \times 6 \mathrm{~mm}^{3}$.

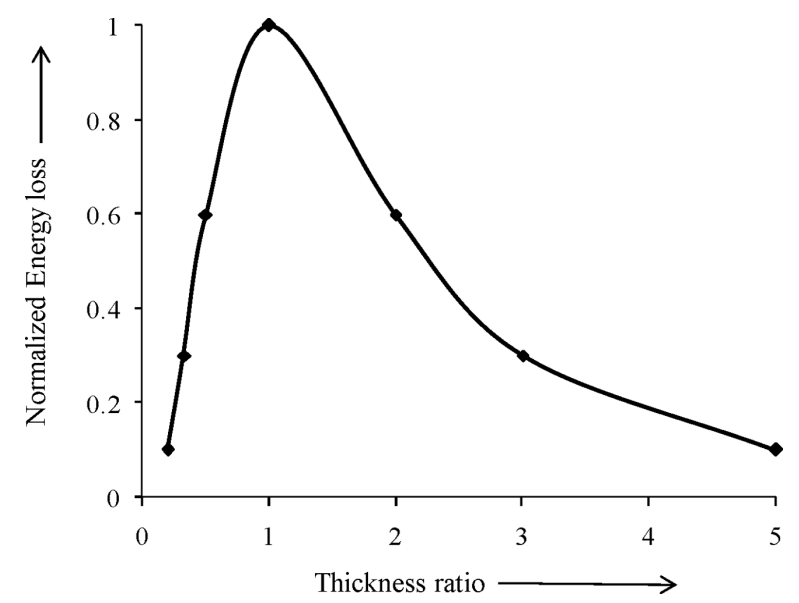

Fig. 5. Variation of normalized energy loss with thickness ratio for welded cantilever beams.

Moreover, the relative slip is maximum at thickness ratio of one and decreases as the ratio is increased. In welded beams of equal thickness, the slip surface is at the centroid of the total beam cross-section. It is evident from the expression (25) that for the beam with the same total thickness, the loss coefficient is increased by having laminates of equal thickness. However, for jointed beam with laminates of unequal thickness, the onset of slip is delayed due to 


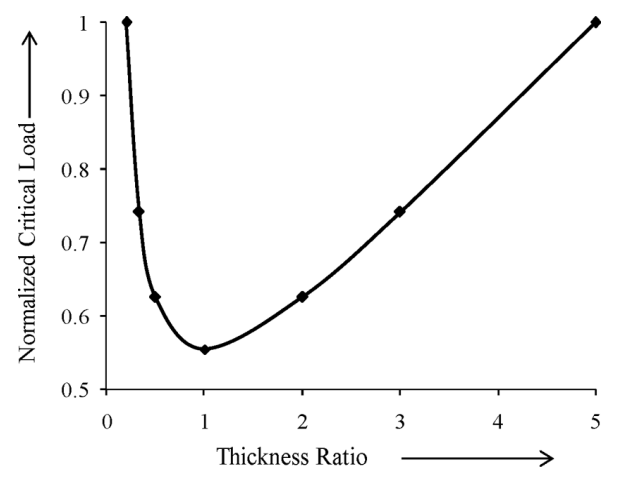

(a)

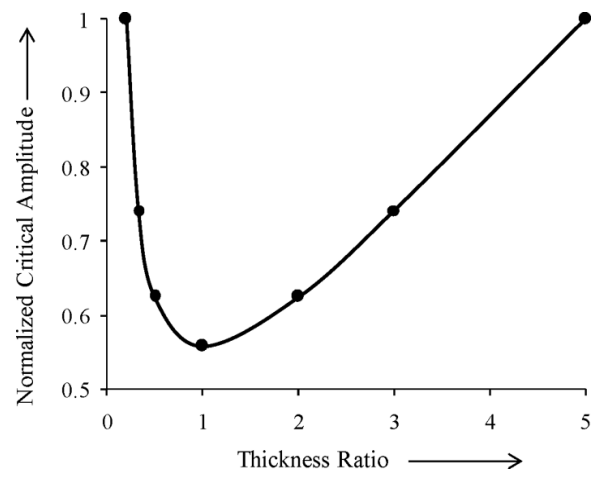

(b)

Fig. 6. (a) Variation of normalized critical load with thickness ratio for welded cantilever beams; (b) Variation of normalized critical amplitude with thickness ratio for welded cantilever beams.

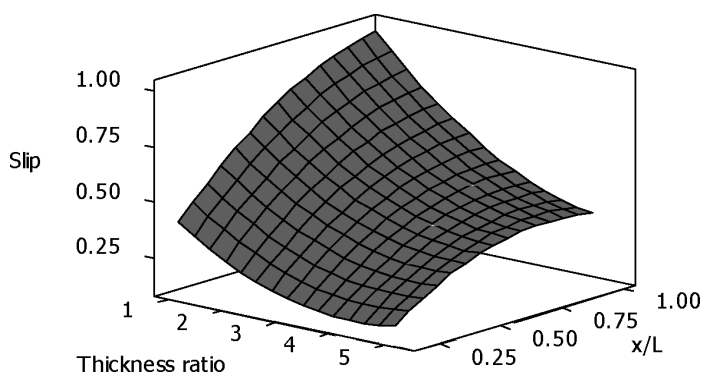

Fig. 7. Surface plot of relative slip with axial position and thickness ratio.

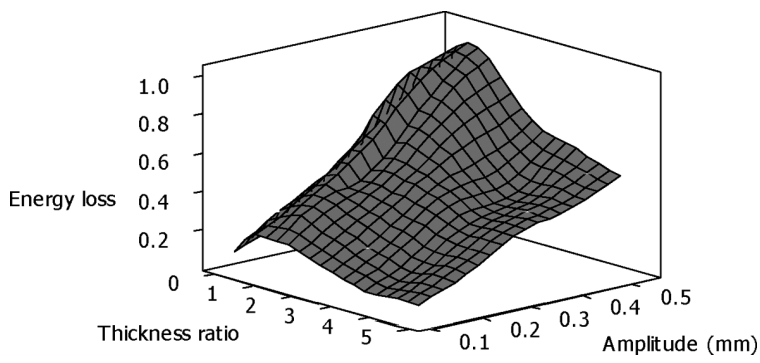

Fig. 8. Surface plot of energy loss with amplitude and thickness ratio.

higher critical load, as compared to that of the laminates of equal thickness as evident from the expression (17). The reason being, slip interface is not at the centroid of the beam in case of layered and welded non-symmetric beams thereby raising the critical load. The energy loss and critical load varies non-linearly with increase in thickness ratio as evident from Figs 5 and 8.

The variation of energy loss with the initial amplitude of excitation for the layered and jointed beams of symmetric and non-symmetric beams has been plotted in Fig. 8. From the figure it is apparent that the energy dissipation increases with the increase in initial amplitude of excitation. From the expressions (19) and (21), it is deduced that the energy loss due to friction is directly proportional to the initial amplitude of excitation which establishes that energy dissipation is enhanced with increase in initial amplitude of excitation. Further, the relative slip at the interfaces is increased due to increase in initial amplitude of excitation as shown in expression (17) thereby enhancing the energy loss due to friction.

The static deflection of the two layered welded beams with various thickness ratios have been shown as a sample in the Figs 9 and 10. From the figure, it is evident that the static deflection decreases with the increase in thickness ratio.

The theoretical loss factor has been evaluated for various configurations and vibration conditions using the expression (25) as well as the corresponding experimental ones are plotted as solid ( - ) and dotted lines ( - ) in Figs 11 to 13 respectively for comparison. It is observed that both the curves are in good agreement with a maximum variation of $7.8 \%$ which shows the authenticity of the theoretical analysis.

Loss factor decreases with the increase in the initial amplitude of excitation at the free end of the beam model although increase in the amplitude of vibration raises energy loss due to friction, as evident from Fig. 11. The strain energy introduced into the system is proportional to square of the amplitude as given by expression (23). However, the increase in amplitude of excitation increases the input strain energy at a higher rate compared to the energy loss due to friction which is linearly proportional to the initial amplitude as given by expressions (19) and (21), thereby reducing the damping capacity. 


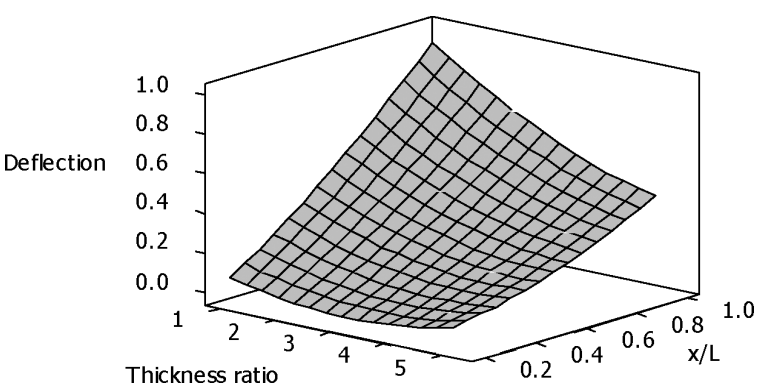

Fig. 9. Surface plot of static deflection of the welded beam with axial position and thickness ratio greater than one.

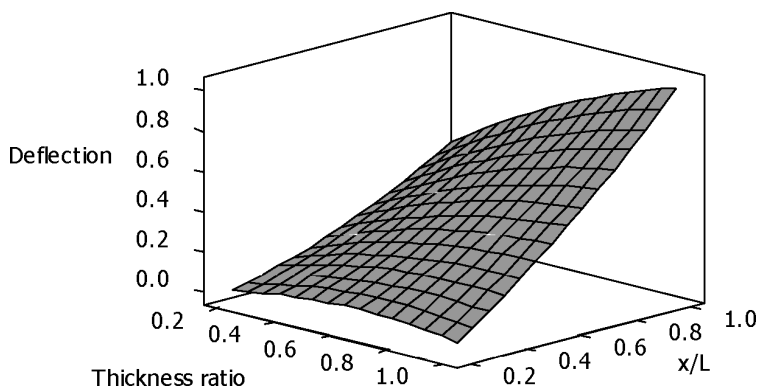

Fig. 10. Surface plot of static deflection of the welded beam with axial position and thickness ratio less than one.

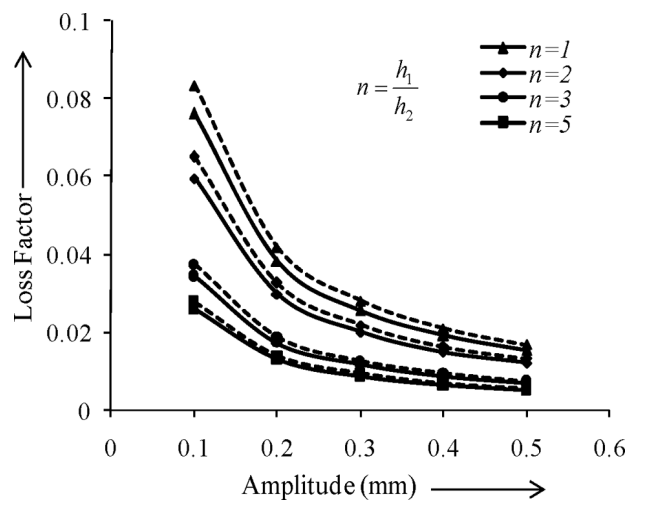

(a)

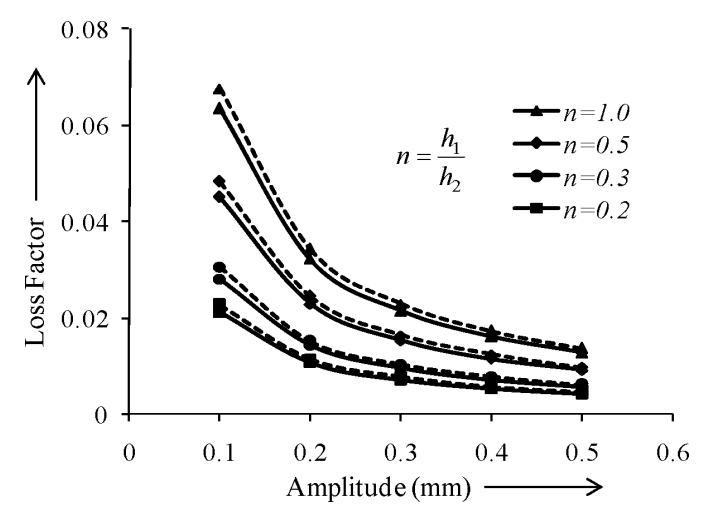

(b)

Fig. 11. (a) Variation of loss factor with amplitude for jointed and welded cantilever beams of dimensions $=600.6 \times 40.2 \times 6 \mathrm{~mm}^{3}$; (b) Variation of loss factor with amplitude for jointed and welded cantilever beams of dimensions $=560.2 \times 40.2 \times 6 \mathrm{~mm}^{3}$.

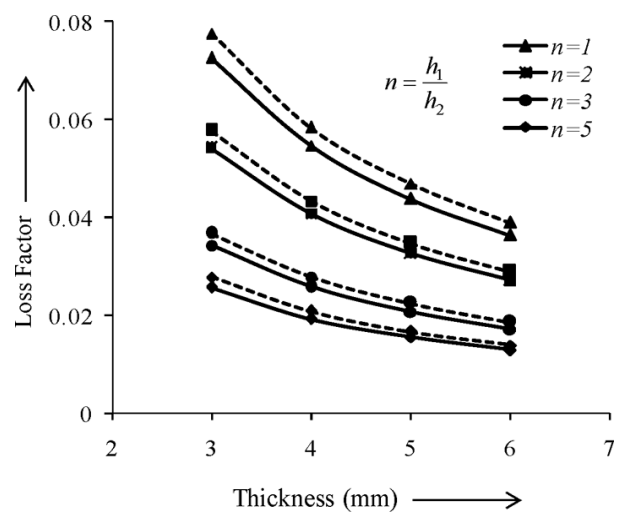

(a)

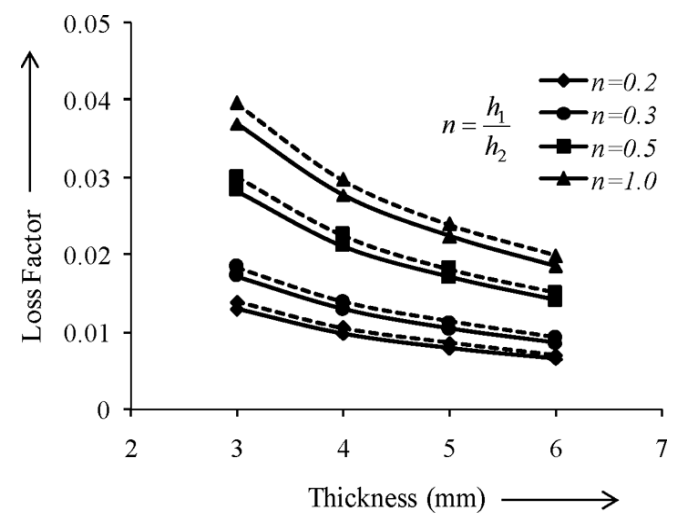

(b)

Fig. 12. (a) Variation of loss factor with thickness for jointed and welded cantilever beams of dimensions $=600.6 \times 40.2 \mathrm{~mm}^{2}$ and amplitude $=$ $0.1 \mathrm{~mm}$; (b) Variation of loss factor with thickness for jointed and welded cantilever beams of dimensions $=560.2 \times 40.2 \mathrm{~mm}^{2}$ and amplitude $=0.2 \mathrm{~mm}$.

From the Fig. 12, it is concluded that the damping capacity decreases with the increase in thickness. From the expression (23), it is evident that the strain energy introduced in to the system is enhanced with the increase in thickness. Expression (17) reveals that the increase in thickness increases the relative slip thereby raising the energy loss. Although the energy loss is enhanced with the increase in thickness, the damping capacity is reduced as the dissipation of energy is at a slower rate compared to that of the input strain energy. 


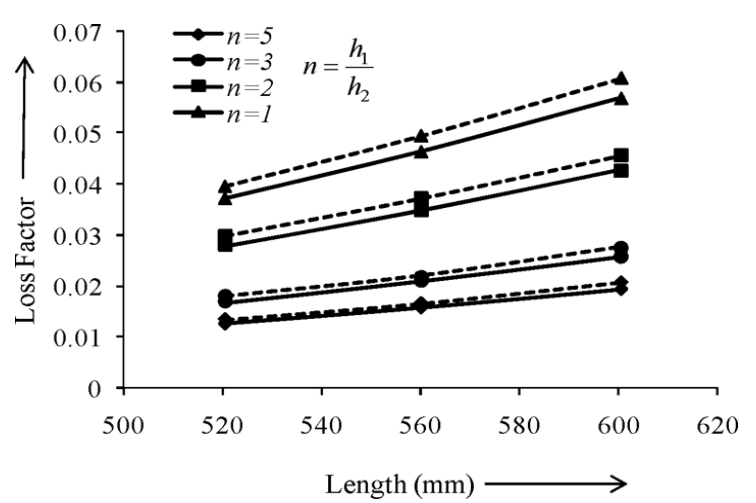

(a)

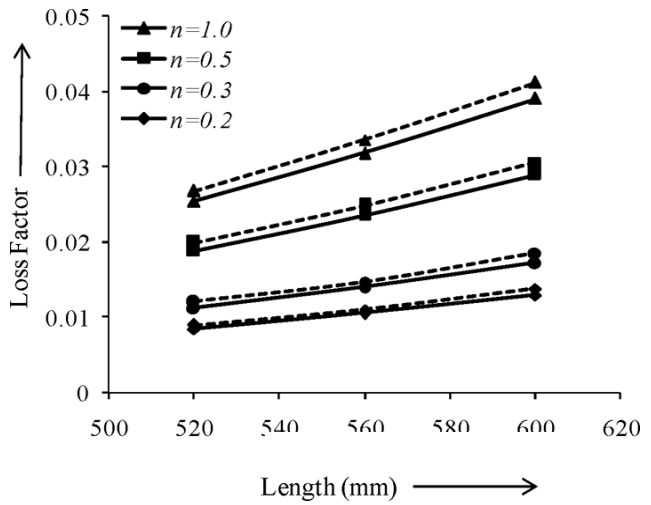

(b)

Fig. 13. (a) Variation of loss factor with length for jointed and welded cantilever beams of dimensions $=40.2 \times 6 \mathrm{~mm}^{2}$ and amplitude $=0.1 \mathrm{~mm}$; (b) Variation of loss factor with length for jointed and welded cantilever beams of dimensions $=40.2 \times 6 \mathrm{~mm}^{2}$ and amplitude $=0.2 \mathrm{~mm}$.

Damping capacity of the layered and welded structures increases with the increase in length as shown in Fig. 13. With the increase in length, the interface area is increased resulting in greater dissipation of the energy due to friction. Furthermore, with increase in the length of the jointed beam, strain energy introduced into the system is reduced as evident from the expression (23). Hence, the overall effect is an increase in the damping capacity of the system.

\section{Summary and conclusion}

In the present work, the mechanism of slip damping in layered and welded structures with unequal thickness has been proposed. Proposed mathematical model for damping is validated by conducting experiments. A set of measurements have been taken, which served as a qualitative, experimental validation of the procedure. It is found that the damping capacity of layered and welded structures is substantially enhanced by fabricating the structures with symmetric beams. It is also observed that the onset of slip is delayed in the structures fabricated with beams of unequal thickness. Energy dissipation is maximized by having the slip interface at the centroid of the total beam cross-section which is true in case of jointed beams of equal thickness. Further, it is also deduced that the pressure distribution characteristics, relative slip and kinematic co-efficient of friction at the interfaces, length, thickness, and initial amplitude of excitation are the vital parameters influencing the damping capacity of fabricated structures with unequal thickness. The present analysis can further be extended to include the laminates of different materials. This investigation will help the designers to model the complicated realistic structures in which slip damping is predominant.

\section{References}

[1] L.E. Goodman and J.H. Klumpp, Analysis of Slip Damping With Reference to Turbine Blade Vibration, Journal of Applied Mechanics 23 (1956), 421.

[2] C. Cockerham and G.R. Symmons, Stability criterion for stick-slip motion using a discontinuous dynamic friction model, Wear 40 (1976), $113-120$.

[3] D.P. Hess et al., Normal vibrations and friction at a Hertzian contact under random excitation: theory and experiment, Journal of Sound and Vibration 153(3) (1992), 491-508.

[4] A. Guyan et al., Dynamic with Friction, Modeling, Analysis and experiment, Series on Stability, Vibration and Control of Systems, World Scientific, Singapore, 1996.

[5] D.M. Barnett et al., Slip wave along the interface between two anisotropic elastic half-space in sliding contact, Proceedings of the Royal Society of London, Series A 415 (1988), 389-419.

[6] G.A. Maugin et al., Interfacial waves in the presence of areas of slip, Geophysical Journal International 118 (1994), 305-316.

[7] L.E. Goodman, A Review of Progress in Analysis of Interfacial Slip Damping, Structural Damping, J. Ruzika, ed., (ASME), New York, 1959. 
[8] S.W.E. Earles, Theoretical Estimation of the Frictional Energy Dissipation in a Simple Lap Joint, Journal of Mechanical Engineering Sciences 8 (1966), 207.

[9] A.S.R. Murty, On Damping of Thin Cantilevers, Ph.D. Thesis, Department of Mechanical Engineering, I. I. T., Kharagpur, 1971.

[10] M. Masuko, Y. Ito and K. Yoshida, Theoretical analysis for a damping ratio of jointed cantibeam, Bulletin of JSME 16 (1973), $1421-1432$.

[11] N. Nishiwaki, M. Masuko, Y. Ito and I. Okumura, A study on damping capacity of a jointed cantilever beam (1st Report; Experimental Results), Bulletin of JSME 21 (1978), 524-531.

[12] M. Motosh, Stress distribution in Joints of bolted or riveted connections, Transactions of ASME, Journal of Engineering for Industry, 1975.

[13] S.W. Hansen and R. Spies, Structural Damping in Laminated Beams Due to Interfacial Slip, Journal of Sound and Vibration 204(2) (1997), 183-202.

[14] B.K. Nanda and A.K. Behera, Study on Damping in Layered and Jointed Structures with Uniform Pressure Distribution at the Interfaces, Journal of Sound and Vibration 226(4) (1999), 607-624.

[15] H.H. Ziada and A.K. Abd, Load pressure distribution and contact areas in bolted joints, Institution of Engineers (India) 61 (1980), $93-100$.

[16] B.K. Nanda and A.K. Behera, Damping in layered and jointed structures, International Journal of Acoustics and Vibration 5(2) (2000), 89-95.

[17] B.K. Nanda and A.K. Behera, Improvement of damping capacity of structural members using layered construction, Proceedings of Seventh International Congress on Sound and Vibration, Garmisch-Partenkirchen, Germany, 2000, pp. 3059-3066.

[18] B.K. Nanda, Study of the effect of bolt diameter and washer on damping in layered and jointed structures, Journal of Sound and Vibration 290 (2006), 1290-1314.

[19] O. Damisa, V.O.S. Olunloyo, C.A. Osheku and A.A. Oyediran, Static analysis of slip damping with clamped laminated beams, European Journal of Scientific Research 17(4) (2007), 455-476.

[20] O. Damisa, V.O.S. Olunloyo, C.A. Osheku and A.A. Oyediran, Dynamic Analysis of Slip Damping in Clamped Layered Beams With Non-Uniform Pressure Distribution at the Interface, Journal of Sound and Vibration 309 (2008), 349-374.

[21] B. Singh and B.K. Nanda, Effect of welding on the slip damping of layered and jointed structures, Journal of Engineering Mechanics, ASCE 136(7) (2010), 928-932.

[22] M. Ciavarella, D.A. Hills and G. Monno, The influence of rounded edges on indentation by flat punch, Proceedings Inst Mech Eng, Part C: J Mech Eng Sci 212 (1998), 319-327.

[23] K.L. Johnson, Contact Mechanics, Cambridge University Press, New York, 1985.

[24] A.E. Giannakopoulos, T.C. Lindley, S. Suresh and C. Chenut, Similarities of stress concentrations in contact at round punches and fatigue at notches: Implications to fretting fatigue crack initiation, Fatigue Fract Eng Mater Struct 23(7) (2000), 561-571.

[25] G.B. Warburton, The Dynamical Behaviour of Structures. 2nd ed., Pergamon Press Ltd., Oxford, England, 1976, pp. 204-205, $230-231$. 

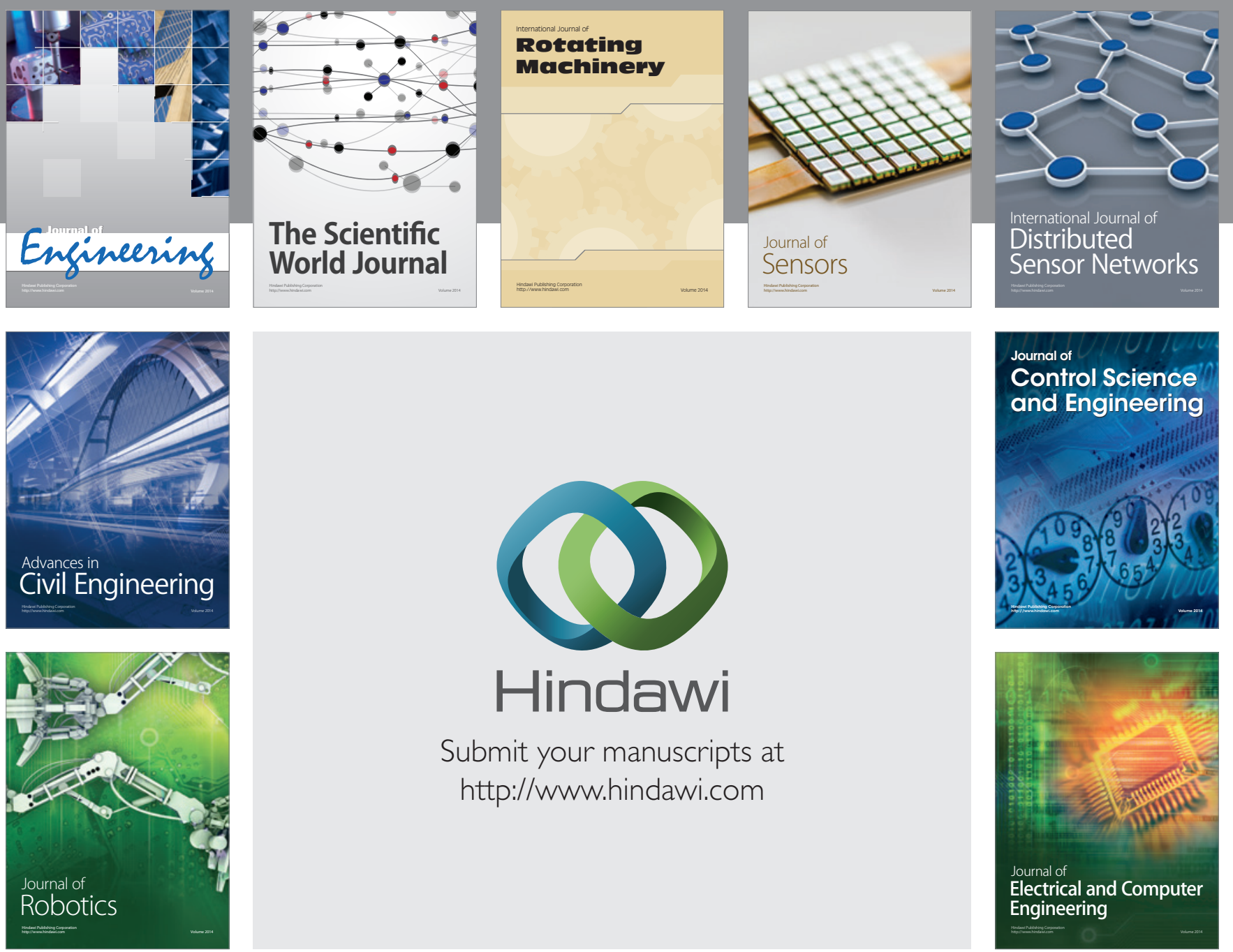

Submit your manuscripts at

http://www.hindawi.com
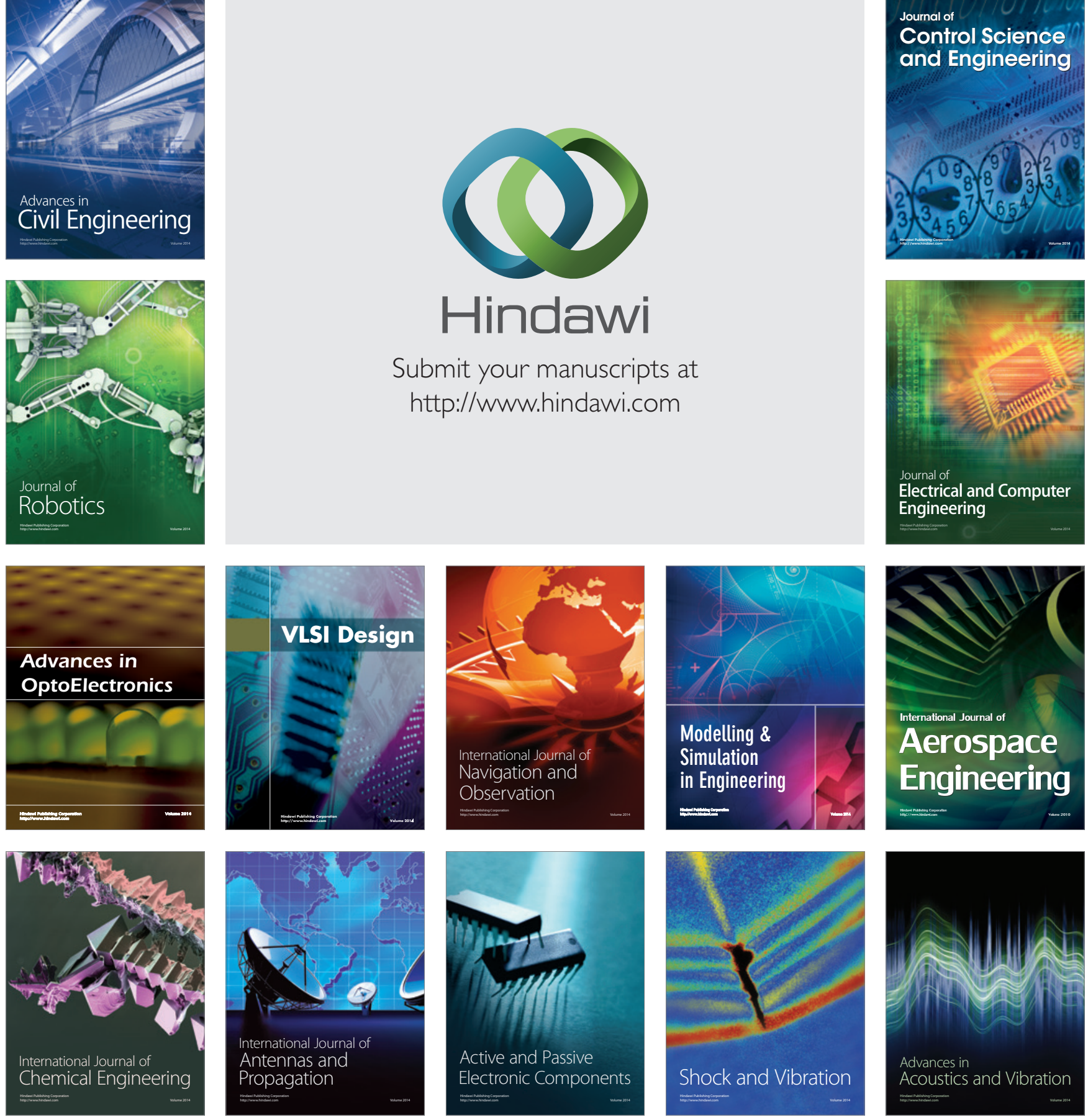